\title{
A New Stability Criterion for Systems with Distributed Time-Varying Delays via Mixed Inequalities Method
}

\author{
Jun-kang Tian $(\mathbb{D})$ and Yan-min Liu \\ School of Mathematics, Zunyi Normal University, Zunyi, Guizhou 563006, China \\ Correspondence should be addressed to Yan-min Liu; yanminliuzunyi@163.com
}

Received 29 January 2020; Accepted 25 March 2020; Published 14 April 2020

Academic Editor: Xinzhi Liu

Copyright (c) 2020 Jun-kang Tian and Yan-min Liu. This is an open access article distributed under the Creative Commons Attribution License, which permits unrestricted use, distribution, and reproduction in any medium, provided the original work is properly cited.

This paper is concerned with the delay-dependent stability of systems with distributed time-varying delays. The novelty relies on the use of some new inequalities which are less conservative than some existing inequalities. A less conservative stability criterion is obtained by constructing some new augmented Lyapunov-Krasovskii functionals, which are given in terms of linear matrix inequalities. The effectiveness of the presented criterion is demonstrated by two numerical examples.

\section{Introduction}

Consider the systems with distributed time-varying delays:

$$
\begin{aligned}
\dot{x}(t) & =A x(t)+B x(t-h(t))+C \int_{t-h(t)}^{t} x(s) \mathrm{d} s, \\
x(t) & =\phi(t), \\
t & \in[-h, 0],
\end{aligned}
$$

where $x(t) \in R^{n}$ is the system state, $A, B, C \in R^{n \times n}$ are constant matrices, and $h(t)$ is the time-varying delay satisfying

$$
0 \leq h(t) \leq h, \quad-u \leq \dot{h}(t) \leq u<1
$$

Since time delays occur in many dynamic systems, stability analysis of the time delay system [1-5] has become a hot topic in the past few decades. Due to the representation of linear systems with time-varying delays, the delaydependent stability analysis via the LKF method has attracted much attention. The conservatism of the LKF method comes from two aspects: the construction of the LKF and the bound on its derivative. Selecting the LKF is crucial to derive less conservative criteria. An augmented LKF [6] is proposed to reduce the conservatism in the early literature. Recently, a new augmented LKF [7] is introduced by employing the information of a second-order Bessel-Legendre inequality. It is necessary to take the derivative of the LKF to derive a stability criterion. The difficulty lies in the bounds of the integrals that arise in the derivative of the LKF. There are two main methods for dealing with such integrals: the free-weighting matrix method [8] and the integral inequality method. The integral inequality method includes various integral inequalities, such as Jensen inequality [9-11], Wirtinger-based inequality [12-15], free matrix-based inequality $[16,17]$, auxiliary function-based inequality [18], relaxed integral inequality [19], and Bessel-Legendre inequality [20]. Very recently, the improved inequality-based functions approach [21] is proposed to derive less conservative results for systems with time-varying delays. However, when estimating $\dot{V}\left(x_{t}\right), \int_{a}^{b} \dot{x}^{T}(s) R \dot{x}(s) \mathrm{d} s$ is only estimated as $\int_{a}^{b} \dot{x}^{T}(s) R \dot{x}(s) \mathrm{d} s \geq(1 / b-a) \Omega_{1}^{T} R \Omega_{1}+(3 / b-a) \Omega_{2}^{T} R \Omega_{2}+$ $(5 / b-a) \Omega_{3}^{T} R \Omega_{3} . \Omega_{1}, \Omega_{2}$, and $\Omega_{3}$ are the same as in Lemma 2. Then, a new integral inequality was proposed in [22] to 
further reduce the conservatism. But the integral inequality can only deal with the constant time delay. On the other hand, stability analysis for systems with distributed delays is of both practical and theoretical importance. Then, it is desirable to extend the system model to include distributed delays. In recent years, the stability analysis of systems with distributed delays has been received considerable attention [23-27]. But only the authors in [25, 26] consider the systems with distributed time-varying delays.

This paper is concerned with the delay-dependent stability of systems with distributed time-varying delays. Based on some new inequalities and some new augmented LKFs, a less conservative stability criterion is obtained in terms of LMIs. Our paper has two characteristics: (1) the integral $\int_{a}^{b} \dot{x}^{T}(s) R \dot{x}(s) \mathrm{d} s$ is estimated as $\int_{a}^{b} \dot{x}^{T}(s) R \dot{x}(s) \mathrm{d} s \geq(1 / b-$ a) $\Omega_{1}^{T} R \Omega_{1}+(3 / b-a) \Omega_{2}^{T} R \Omega_{2}+(5 / b-a) \Omega_{3}^{T} R \Omega_{3}+(7 / b-a)$ $\Omega_{4}^{T} R \Omega_{4}$, which includes those in $[9,13,20]$ as special cases. $\Omega_{i}, i=1,2,3,4$, is the same as in Lemma 2. (2) An augmented LKF which contains more information about $h(t)$ is proposed to reduce the conservatism. The effectiveness of the presented criterion is demonstrated by two numerical examples.

Throughout this paper, the set $S^{n}$ denotes the set of symmetric matrices and the set $S_{+}^{n}$ denotes the set of symmetric positive definite matrices. For any square matrix $P$, we define $\operatorname{Sym}(P)=P+P^{T}$.

\section{Main Results}

In this section, the following lemmas are introduced to derive the main results.

Lemma 1 (see [20]). For any matrices $\Theta \in S_{+}^{n}$, $M_{1}, M_{2} \in R^{m \times n}, Y \in R^{2 n \times m}$, and $\forall \alpha \in(0,1)$, the inequality

$$
\begin{array}{r}
-\Upsilon^{T}\left[\begin{array}{cc}
\frac{1}{\alpha} \Theta & 0 \\
0 & \frac{1}{1-\alpha} \Theta
\end{array}\right] \Upsilon \leq \\
\\
+\alpha M_{1} \Theta^{-1} M_{1}^{T}+(1-\alpha) \Upsilon_{2}^{T} \sum(\alpha) \Upsilon-\operatorname{Sym}\left(\Upsilon^{T}\left[\begin{array}{c}
(1-\alpha) M_{1}^{T} \\
\alpha M_{2}^{T}
\end{array}\right]\right)
\end{array}
$$

holds, where

$$
\Sigma(\alpha)=\left[\begin{array}{cc}
(2-\alpha) \Theta & 0 \\
0 & (1+\alpha) \Theta
\end{array}\right]
$$

Lemma 2 (see [22]). For a matrix $R \in S_{+}^{n}$ and any continuously differentiable function $x:[a, b] \longrightarrow R^{n}$, the inequality

$$
\begin{aligned}
\int_{a}^{b} \dot{x}^{T}(s) R \dot{x}(s) \mathrm{d} s \geq & \frac{1}{b-a} \Omega_{1}^{T} R \Omega_{1}+\frac{3}{b-a} \Omega_{2}^{T} R \Omega_{2} \\
& +\frac{5}{b-a} \Omega_{3}^{T} R \Omega_{3}+\frac{7}{b-a} \Omega_{4}^{T} R \Omega_{4},
\end{aligned}
$$

holds, where

$$
\begin{aligned}
\Omega_{1}= & x(b)-x(a), \\
\Omega_{2}= & x(b)+x(a)-\frac{2}{b-a} \int_{a}^{b} x(s) \mathrm{d} s, \\
\Omega_{3}= & x(b)-x(a)+\frac{6}{b-a} \int_{a}^{b} x(s) \mathrm{d} s-\frac{12}{(b-a)^{2}} \int_{a}^{b} \int_{u}^{b} x(s) \mathrm{d} s \mathrm{~d} u, \\
\Omega_{4}= & x(b)+x(a)-\frac{12}{b-a} \int_{a}^{b} x(s) \mathrm{d} s+\frac{60}{(b-a)^{2}} \int_{a}^{b} \int_{u}^{b} x(s) \mathrm{d} s \mathrm{~d} u \\
& -\frac{120}{(b-a)^{3}} \int_{a}^{b} \int_{u}^{b} \int_{v}^{b} x(s) \mathrm{d} s \mathrm{~d} v \mathrm{~d} u .
\end{aligned}
$$

Lemma 3 (see [28]). Suppose that $\Omega, \Omega_{i j}(i, j=1,2)$ are the constant matrices of appropriate dimensions, $\alpha \in[0,1]$, $\beta \in[-u, u], 0 \leq u<1$, then

$$
\Omega+\alpha \Omega_{11}+(1-\alpha) \Omega_{12}+\beta \Omega_{21}+(1-\beta) \Omega_{22}<0,
$$

holds if and only if the following inequalities hold:

$$
\begin{aligned}
& \Omega+\Omega_{11}-u \Omega_{21}+(1+u) \Omega_{22}<0, \\
& \Omega+\Omega_{12}-u \Omega_{21}+(1+u) \Omega_{22}<0, \\
& \Omega+\Omega_{11}+u \Omega_{21}+(1-u) \Omega_{22}<0, \\
& \Omega+\Omega_{12}+u \Omega_{21}+(1-u) \Omega_{22}<0 .
\end{aligned}
$$

Based on Lemmas 1-3, a novel stability criterion is derived for system (1) with distributed time-varying delays.

Theorem 1. For given scalars $h>0, u>0$, if there exist matrices $P, Q_{1}, Q_{2}, \in S_{+}^{2 n}, Q_{3} \in S_{+}^{n}, M_{1}, M_{2} \in R^{10 n \times 4 n}$, such that the LMI

$$
\Phi(\alpha, \beta)=\left[\begin{array}{cc}
\phi(\alpha, \beta)-\Upsilon^{T} \Sigma(\alpha) \Upsilon-\operatorname{Sym}\left(\Upsilon^{T}\left[\begin{array}{c}
(1-\alpha) M_{1}^{T} \\
\alpha M_{2}^{T}
\end{array}\right]\right) & * \\
\alpha M_{1}^{T}+(1-\alpha) M_{2}^{T} & -\Theta
\end{array}\right]<0,
$$

holds for $\alpha=\{0,1\}, \dot{h}(t)=\beta=\{-u, u\}$, i.e.,

$$
\begin{gathered}
\Phi(0,-u)<0, \\
\Phi(0, u)<0, \\
\Phi(1,-u)<0, \\
\Phi(1, u)<0,
\end{gathered}
$$

then, system (1) is asymptotically stable, where 


$$
\begin{aligned}
\phi(\alpha, \beta)= & S y m\left(\Pi_{1}^{T} P \Pi_{2}\right)+\Pi_{3}^{T} Q_{1} \Pi_{3} \\
& -(1-\beta) \Pi_{4}^{T} Q_{1} \Pi_{4}+\operatorname{Sym}\left(\Pi_{5}^{T} Q_{2} \Pi_{6}\right)+h^{2} \varepsilon_{0}^{T} Q_{3} \varepsilon_{0}, \\
\Pi_{1}= & {\left[\begin{array}{ll}
\varepsilon_{1}^{T} & \alpha h \varepsilon_{5}^{T}+(1-\alpha) h \varepsilon_{6}^{T}
\end{array}\right]^{T}, } \\
\Pi_{2}= & {\left[\begin{array}{ll}
A \varepsilon_{1}^{T}+B \varepsilon_{2}^{T}+C \alpha h \varepsilon_{5}^{T} & \varepsilon_{1}^{T}-\varepsilon_{3}^{T}
\end{array}\right]^{T}, } \\
\Pi_{3}= & {\left[\begin{array}{ll}
\varepsilon_{1}^{T} & A \varepsilon_{1}^{T}+B \varepsilon_{2}^{T}+C \alpha h \varepsilon_{5}^{T}
\end{array}\right]^{T}, } \\
\Pi_{4}= & {\left[\begin{array}{ll}
\varepsilon_{2}^{T} & \varepsilon_{4}^{T}
\end{array}\right]^{T}, } \\
\Pi_{5}= & {\left[\begin{array}{ll}
\varepsilon_{1}^{T} & \varepsilon_{2}^{T}
\end{array}\right]^{T}, } \\
\Pi_{6}= & {\left[A \varepsilon_{1}^{T}+B \varepsilon_{2}^{T}+C \alpha h \varepsilon_{5}^{T} \quad(1-\beta) \varepsilon_{4}^{T}\right]^{T}, } \\
\Pi_{7}= & \varepsilon_{1}-\varepsilon_{2}, \\
\Pi_{8}= & \varepsilon_{1}+\varepsilon_{2}-2 \varepsilon_{5}, \\
\Pi_{9}= & \varepsilon_{1}-\varepsilon_{2}+6 \varepsilon_{5}-12 \varepsilon_{7}, \\
\Pi_{10}= & \varepsilon_{1}+\varepsilon_{2}-12 \varepsilon_{5}+60 \varepsilon_{7}-120 \varepsilon_{9}, \\
\Pi_{11}= & \varepsilon_{2}-\varepsilon_{3}, \\
\Pi_{12}= & \varepsilon_{2}+\varepsilon_{3}-2 \varepsilon_{6}, \\
\Pi_{13}= & \varepsilon_{2}-\varepsilon_{3}+6 \varepsilon_{6}-12 \varepsilon_{8}, \\
\Pi_{14}= & \varepsilon_{2}+\varepsilon_{3}-12 \varepsilon_{6}+60 \varepsilon_{8}-120 \varepsilon_{10}, \\
\varepsilon_{0}= & A \varepsilon_{1}+B \varepsilon_{2}+C \alpha h \varepsilon_{5}, \\
\Upsilon= & {\left[\Pi_{7}^{T} \Pi_{8}^{T} \quad \Pi_{9}^{T} \Pi_{10}^{T} \Pi_{11}^{T} \Pi_{12}^{T} \Pi_{13}^{T} \Pi_{14}^{T}\right]^{T}, } \\
\Theta= & d i a g\left(Q_{3}, 3 Q_{3}, 5 Q_{3}, 7 Q_{3}\right),
\end{aligned}
$$

and $\varepsilon_{i} \in \mathscr{R}^{n \times 10 n}$ is defined as $\varepsilon_{i}=\left[\begin{array}{lll}0_{n \times(i-1) n} & I_{n} & 0_{n \times(10-i) n}\end{array}\right]$ for $i=1,2, \ldots, 10$.

Proof. Introduce an LKF candidate as

$$
V\left(x_{t}\right)=V_{1}\left(x_{t}\right)+V_{2}\left(x_{t}\right)+V_{3}\left(x_{t}\right)+V_{4}\left(x_{t}\right),
$$

where

$$
\begin{aligned}
& V_{1}\left(x_{t}\right)=\left[\begin{array}{c}
x(t) \\
\int_{t-h}^{t} x(s) d s
\end{array}\right]^{T} P\left[\begin{array}{c}
x(t) \\
\int_{t-h} x(s) d s
\end{array}\right], \\
& V_{2}\left(x_{t}\right)=\int_{t-h(t)}^{t}\left[\begin{array}{c}
x(s) \\
\dot{x}(s)
\end{array}\right]^{T} Q_{1}\left[\begin{array}{c}
x(s) \\
\dot{x}(s)
\end{array}\right] \mathrm{d} s, \\
& V_{3}\left(x_{t}\right)=\left[\begin{array}{c}
x(t) \\
x(t-h(t))
\end{array}\right]^{T} Q_{2}\left[\begin{array}{c}
x(t) \\
x(t-h(t))
\end{array}\right], \\
& V_{4}\left(x_{t}\right)=h \int_{t-h}^{t} \int_{u}^{t} \dot{x}^{T}(s) Q_{3} \dot{x}(s) \mathrm{d} s \mathrm{~d} u .
\end{aligned}
$$

Calculate the derivative of $V\left(x_{t}\right)$ along the solution of system (1) as follows:

$$
\begin{aligned}
& \dot{V}_{1}\left(x_{t}\right)=2\left[\begin{array}{c}
x(t) \\
\int_{t-h}^{t} x(s) \mathrm{d} s
\end{array}\right]^{T} P\left[\begin{array}{c}
\dot{x}(t) \\
x(t)-x(t-h)
\end{array}\right] \\
& =2\left[\begin{array}{c}
x(t) \\
\int_{t-h}^{t} x(s) \mathrm{d} s
\end{array}\right]^{T} P\left[\begin{array}{c}
A x(t)+B x(t-h(t))+C \int_{t-h(t)}^{t} x(s) \mathrm{d} s \\
x(t)-x(t-h)
\end{array}\right], \\
& \dot{V}_{2}\left(x_{t}\right)=\left[\begin{array}{c}
x(t) \\
\dot{x}(t)
\end{array}\right]^{T} Q_{1}\left[\begin{array}{c}
x(t) \\
\dot{x}(t)
\end{array}\right]-(1-\dot{h}(t))\left[\begin{array}{c}
x(t-h(t)) \\
\dot{x}(t-h(t))
\end{array}\right]^{T} Q_{1}\left[\begin{array}{c}
x(t-h(t)) \\
\dot{x}(t-h(t))
\end{array}\right] \\
& =\left[\begin{array}{c}
x(t) \\
A x(t)+B x(t-h(t))+C \int_{t-h(t)}^{t} x(s) \mathrm{d} s
\end{array}\right]^{T} Q_{1}\left[\begin{array}{c}
x(t) \\
A x(t)+B x(t-h(t))+C \int_{t-h(t)}^{t} x(s) \mathrm{d} s
\end{array}\right] \\
& -(1-\dot{h}(t))\left[\begin{array}{c}
x(t-h(t)) \\
\dot{x}(t-h(t))
\end{array}\right]^{T} Q_{1}\left[\begin{array}{c}
x(t-h(t)) \\
\dot{x}(t-h(t))
\end{array}\right] \\
& \dot{V}_{3}\left(x_{t}\right)=2\left[\begin{array}{c}
x(t) \\
x(t-h(t))
\end{array}\right]^{T} Q_{2}\left[\begin{array}{c}
\dot{x}(t) \\
(1-\dot{h}(t)) \dot{x}(t-h(t))
\end{array}\right] \text {, }
\end{aligned}
$$




$$
\begin{gathered}
=2\left[\begin{array}{c}
x(t) \\
x(t-h(t))
\end{array}\right]^{T} Q_{2}\left[\begin{array}{c}
A x(t)+B x(t-h(t))+C \int_{t-h(t)}^{t} x(s) \mathrm{d} s \\
(1-\beta) \dot{x}(t-h(t))
\end{array}\right], \\
\dot{V}_{4}\left(x_{t}\right)=h^{2} \dot{x}^{T}(t) Q_{3} \dot{x}(t)-h \int_{t-h}^{t} \dot{x}^{T}(s) Q_{3} \dot{x}(s) \mathrm{d} s .
\end{gathered}
$$

According to (18)-(22), we can obtain where

$$
\begin{aligned}
\dot{V}\left(x_{t}\right)= & \zeta^{T}(t)\left\{\operatorname{sym}\left(\Pi_{1}^{T} P \Pi_{2}\right)+\Pi_{3}^{T} Q_{1} \Pi_{3}-(1-\beta) \Pi_{4}^{T} Q_{1} \Pi_{4}\right. \\
& \left.+\operatorname{Sym}\left(\Pi_{5}^{T} Q_{2} \Pi_{6}\right)+h^{2} \varepsilon_{0}^{T} Q_{3} \varepsilon_{0}\right\} \zeta(t) \\
& -h \int_{t-h}^{t} \dot{x}^{T}(s) Q_{3} \dot{x}(s) \mathrm{d} s,
\end{aligned}
$$

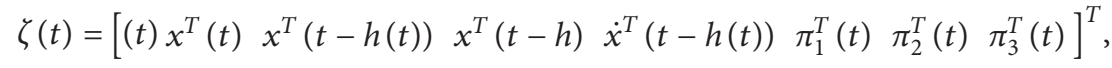

$$
\begin{aligned}
& \pi_{1}(t)=\left[\frac{1}{h(t)} \int_{t-h(t)}^{t} x^{T}(s) \mathrm{d} s \frac{1}{h-h(t)} \int_{t-h}^{t-h(t)} x^{T}(s) \mathrm{d} s\right]^{T}, \\
& \pi_{2}(t)=\left[\frac{1}{h(t)^{2}} \int_{t-h(t)}^{t} \int_{u}^{t} x^{T}(s) \mathrm{d} s \mathrm{~d} u \frac{1}{(h-h(t))^{2}} \int_{t-h}^{t-h(t)} \int_{u}^{t-h(t)} x^{T}(s) \mathrm{d} s\right]^{T}, \\
& \pi_{3}(t)=\left[\frac{1}{h(t)^{3}} \int_{t-h(t)}^{t} \int_{u}^{t} \int_{v}^{t} x^{T}(s) \mathrm{d} s \mathrm{~d} v \mathrm{~d} u \frac{1}{(h-h(t))^{3}} \int_{t-h}^{t-h(t)} \int_{u}^{t-h(t)} \int_{v}^{t-h(t)} x^{T}(s) \mathrm{d} s \mathrm{~d} v \mathrm{~d} u\right]^{T} .
\end{aligned}
$$

Let $\alpha=(h(t) / h)$, then $1-\alpha=(h-h(t) / h)$, applying Lemma 2 , and we have

$$
\begin{aligned}
& -h \int_{t-h}^{t} \dot{x}^{T}(s) Q_{3} \dot{x}(s) \\
= & -h \int_{t-h(t)}^{t} \dot{x}^{T}(s) Q_{3} \dot{x}(s) \mathrm{d} s-h \int_{t-h}^{t-h(t)} \dot{x}^{T}(s) Q_{3} \dot{x}(s) \mathrm{d} s \\
& \leq-\frac{h}{h(t)} \zeta^{T}(t)\left(\Pi_{7}^{T} Q_{3} \Pi_{7}+3 \Pi_{8}^{T} Q_{3} \Pi_{8}+5 \Pi_{9}^{T} Q_{3} \Pi_{9}+7 \Pi_{10}^{T} Q_{3} \Pi_{10}\right) \zeta(t) \\
& -\frac{h}{h-h(t)} \zeta^{T}(t)\left(\Pi_{11}^{T} Q_{3} \Pi_{11}+3 \Pi_{12}^{T} Q_{3} \Pi_{12}+5 \Pi_{13}^{T} Q_{3} \Pi_{13}+7 \Pi_{14}^{T} Q_{3} \Pi_{14}\right) \zeta(t) \\
= & -\frac{1}{\alpha} \zeta^{T}(t)\left(\Pi_{7}^{T} Q_{3} \Pi_{7}+3 \Pi_{8}^{T} Q_{3} \Pi_{8}+5 \Pi_{9}^{T} Q_{3} \Pi_{9}+7 \Pi_{10}^{T} Q_{3} \Pi_{10}\right) \zeta(t) \\
& -\frac{1}{1-\alpha} \zeta^{T}(t)\left(\Pi_{11}^{T} Q_{3} \Pi_{11}+3 \Pi_{12}^{T} Q_{3} \Pi_{12}+5 \Pi_{13}^{T} Q_{3} \Pi_{13}+7 \Pi_{14}^{T} Q_{3} \Pi_{14}\right) \zeta(t) \\
= & -\zeta^{T}(t) \Upsilon^{T}\left[\begin{array}{cc}
\frac{1}{\alpha} \Theta & 0 \\
0 & \frac{1}{1-\alpha} \Theta
\end{array}\right] \Upsilon \zeta(t) .
\end{aligned}
$$


For any matrices $M_{1}, M_{2} \in R^{10 n \times 4 n}$ and applying Lemma 1 , we can obtain

$$
\begin{aligned}
& -\Upsilon^{T}\left[\begin{array}{cc}
\frac{1}{\alpha} \Theta & 0 \\
0 & \frac{1}{1-\alpha} \Theta
\end{array}\right] \Upsilon \\
& \leq-\Upsilon^{T} \Sigma(\alpha) \Upsilon-\operatorname{Sym}\left(\Upsilon^{T}\left[\begin{array}{c}
(1-\alpha) M_{1}^{T} \\
\alpha M_{2}^{T}
\end{array}\right]\right) \\
& \quad+\alpha M_{1} \Theta^{-1} M_{1}^{T}+(1-\alpha) M_{2} \Theta^{-1} M_{2}^{T}=\eta(\alpha) .
\end{aligned}
$$

From (23)-(26), we get

$$
\dot{V}\left(x_{t}\right) \leq \zeta(t)(\phi(\alpha, \beta)+\eta(\alpha)) \zeta(t) .
$$

By Lemma 2 [20], if the LMI (10) is true for $\alpha=\{0,1\}$, $\beta=\{-u, u\}$, then $\phi(\alpha, \beta)+\eta(\alpha)<0$ holds for all $\alpha \in(0,1)$ and $\beta \in[-u, u]$. By Lemma 3, LMI (10) holds if and only if LMIs (11)-(14) hold. This completes the proof.

Remark 1. The integral $\int_{a}^{b} \dot{x}^{T}(s) R \dot{x}(s) \mathrm{d} s$ in $[9,13,20]$ is estimated as $\int_{a}^{b} \dot{x}^{T}(s) R \dot{x}(s) \mathrm{d} s \geq(1 / b-a) \Omega_{1}^{T} R \Omega_{1}, \int_{a}^{b} \dot{x}^{T}(s)$ $R \dot{x}(s) \mathrm{d} s \geq(1 / b-a) \Omega_{1}^{T} R \Omega_{1}+(3 / b-a) \Omega_{2}^{T} R \Omega_{2}, \int_{a}^{b} \dot{x}^{T}(s) R \dot{x}$ $(s) \mathrm{d} s \geq(1 / b-a) \Omega_{1}^{T} R \Omega_{1}+(3 / b-a) \Omega_{2}^{T} R \Omega_{2}+(5 / b-a) \Omega_{3}^{T}$ $R \Omega_{3}$, respectively. In this paper, the integral $\int_{a}^{b} \dot{x}^{T}(s) R \dot{x}(s) \mathrm{d} s$ is estimated as $\int_{a}^{b} \dot{x}^{T}(s) R \dot{x}(s) \mathrm{d} s \geq(1 / b-$ a) $\Omega_{1}^{T} R \Omega_{1}+(3 / b-a) \Omega_{2}^{T} R \Omega_{2}+(5 / b-a) \Omega_{3}^{T} R \Omega_{3}+(7 / b-a)$ $\Omega_{4}^{T} R \Omega_{4}$, which includes those in $[9,13,20]$ as special cases. So our method can yield less conservative results.

Remark 2. An augmented LKF which contains more information about time-varying delay $h(t)$ which was proposed to reduce the conservatism. $\dot{x}(t-h(t))$ is added as a state vector, which may yield less conservative criteria.

\section{Numerical Examples}

Two numerical examples are given to demonstrate advantages of the proposed criterion.

Example 1. Consider system (1) with

$$
\begin{aligned}
& A=\left[\begin{array}{cc}
0.0 & 1.0 \\
-1.0 & -2.0
\end{array}\right], \\
& B=\left[\begin{array}{cc}
0.0 & 0.0 \\
-1.0 & 1.0
\end{array}\right], \\
& C=\left[\begin{array}{ll}
0 & 0 \\
0 & 0
\end{array}\right] .
\end{aligned}
$$

For different $u$, Table 1 presents the allowable upper bound of $h(t)$, which guarantees the stability of system (1). Table 1 shows that our method produces the larger upper bound $h$ than those in $[7,12,13,16,17,21]$. In this sense, our
TABLE 1: Upper bound of $h$ for Example 1 with different $u$.

\begin{tabular}{lcccc}
\hline$u$ & 0.1 & 0.2 & 0.5 & 0.8 \\
\hline$[13]$ & 6.590 & 3.672 & 1.411 & 1.275 \\
{$[12]$} & 7.125 & 4.413 & 2.243 & 1.662 \\
{$[16]$} & 7.148 & 4.466 & 2.352 & 1.768 \\
{$[17]$} & 7.167 & 4.517 & 2.415 & 1.838 \\
{$[7]$} & 7.230 & 4.556 & 2.509 & 1.940 \\
{$[21]$} & 7.297 & 4.625 & 2.264 & 2.038 \\
Theorem 1 & 10.095 & 6.808 & 3.676 & 2.615 \\
\hline
\end{tabular}

TABLE 2: Upper bound of $h$ for Example 2 with different $u$.

\begin{tabular}{lcccc}
\hline$u$ & 0.1 & 0.2 & 0.5 & 0.8 \\
\hline$[13]$ & 4.703 & 3.834 & 2.420 & 2.137 \\
{$[11]$} & 4.753 & - & 2.429 & 2.183 \\
{$[16]$} & 4.788 & 4.060 & 3.055 & 2.615 \\
{$[15]$} & 4.93 & 4.22 & 3.09 & 2.66 \\
{$[7]$} & 4.910 & - & 3.233 & 2.789 \\
{$[21]$} & 4.996 & 4.308 & 3.251 & 2.867 \\
Theorem 1 & 5.650 & 4.913 & 3.793 & 3.251 \\
\hline
\end{tabular}

stability criterion is less conservative than those in $[7,12,13,16,17,21]$.

Example 2. Consider system (1) with

$$
\begin{aligned}
& A=\left[\begin{array}{cc}
-2 & 0 \\
0 & -0.9
\end{array}\right], \\
& B=\left[\begin{array}{cc}
-1.0 & 0.0 \\
-1.0 & -1.0
\end{array}\right], \\
& C=\left[\begin{array}{ll}
0 & 0 \\
0 & 0
\end{array}\right] .
\end{aligned}
$$

For different $u$, Table 2 presents the allowable upper bound of $h(t)$, which guarantees the stability of system (1). Table 2 shows that our method produces the larger upper bound $h$ than those in $[7,11,13,15,16,21]$. In this sense, our stability criterion is less conservative than those in $[7,11,13,15,16,21]$.

\section{Conclusions}

This paper focus on delay-dependent stability analysis for systems with distributed time-varying delays. The novelty relies on the use of some new inequalities which are less conservative than some existing inequalities. A less conservative stability criterion is obtained by constructing some new augmented LKFs. The effectiveness of the presented criterion is demonstrated by two numerical examples. In addition, the proposed method can be applied to stability analysis of other dynamic systems such as fuzzy systems with time-varying delay and neutral systems with time-varying delay.

\section{Data Availability}

No additional data are available for this paper. 


\section{Conflicts of Interest}

The authors declare that there are no conflicts of interest.

\section{Acknowledgments}

This work was supported by the Innovative Groups of Education Department of Guizhou Province (Qian jiao he KY [2016]046); High-level Innovative Talents in Guizhou Province (Zun shi ke he rencai [2016]13).

\section{References}

[1] K. Shi, J. Wang, S. Zhong, Y. Tang, and J. Cheng, "Hybriddriven finite-time $H_{\infty}$ sampling synchronization control for coupling memory complex networks with stochastic cyber attacks," Neurocomputing, vol. 387, pp. 241-254, 2020.

[2] K. Shi, J. Wang, S. Zhong, Y. Tang, and J. Cheng, "Non-fragile memory filtering of T-S fuzzy delayed neural networks based on switched fuzzy sampled-data control," Fuzzy Sets and Systems, 2019, In press.

[3] K. Shi, J. Wang, Y. Tang, and S. Zhong, "Reliable asynchronous sampled-data filtering of T-S fuzzy uncertain delayed neural networks with stochastic switched topologies," Fuzzy Sets and Systems, vol. 381, no. 15, pp. 1-25, 2020.

[4] T. Zhao and S. Dian, "State feedback control for interval type2 fuzzy systems with time-varying delay and unreliable communication links," IEEE Transactions on Fuzzy Systems, vol. 26, no. 2, pp. 951-966, 2018.

[5] T. Zhao, M. B. Huang, and S. Y. Dian, "Robust stability and stabilization conditions for nonlinear networked control systems with network-induced delay via T-S fuzzy model," IEEE Transactions Fuzzy Systems, 2019, In press.

[6] Y. He, Q.-G. Wang, L. Xie, and C. Lin, "Further improvement of free-weighting matrices technique for systems with timevarying delay," IEEE Transactions on Automatic Control, vol. 52, no. 2, pp. 293-299, 2007.

[7] X. M. Zhang, Q. L. Han, A. Seuret, and F. Gouaisbaut, "An improved reciprocally convex inequality and an augmented Lyapunov-Krasovskii functional for stability of linear systems with time-varying delay," Automatica, vol. 84, pp. 221-226, 2017.

[8] M. Wu, Y. He, J.-H. She, and G.-P. Liu, "Delay-dependent criteria for robust stability of time-varying delay systems," Automatica, vol. 40, no. 8, pp. 1435-1439, 2004.

[9] K. Gu, "An integral inequality in the stability problem of timedelay systems," in Proceedings of the 39th IEEE Conference on Decision and Control, pp. 2805-2810, Sydney, Australia, 2010.

[10] P. Park, J. W. Ko, and C. Jeong, "Reciprocally convex approach to stability of systems with time-varying delays," Automatica, vol. 47, no. 1, pp. 235-238, 2011.

[11] J.-H. Kim, "Further improvement of Jensen inequality and application to stability of time-delayed systems," Automatica, vol. 64, pp. 121-125, 2016.

[12] O. M. Kwon, M. J. Park, J. H. Park, S. M. Lee, and E. J. Cha, "Improved results on stability of linear systems with timevarying delays via Wirtinger-based integral inequality," Journal of the Franklin Institute, vol. 351, no. 12, pp. 53865398, 2014.

[13] A. Seuret and F. Gouaisbaut, "Wirtinger-based integral inequality: application to time-delay systems," Automatica, vol. 49, no. 9, pp. 2860-2866, 2013.
[14] M. Park, O. Kwon, J. H. Park, S. Lee, and E. Cha, "Stability of time-delay systems via Wirtinger-based double integral inequality," Automatica, vol. 55, pp. 204-208, 2015.

[15] A. Seuret, K. Liu, and F. Gouaisbaut, "Generalized reciprocally convex combination lemmas and its application to time-delay systems," Automatica, vol. 95, pp. 488-493, 2018.

[16] H.-B. Zeng, Y. He, M. Wu, and J. She, "Free-matrix-based integral inequality for stability analysis of systems with timevarying delay," IEEE Transactions on Automatic Control, vol. 60 , no. 10, pp. 2768-2772, 2015.

[17] T. H. Lee, J. H. Park, and S. Xu, "Relaxed conditions for stability of time-varying delay systems," Automatica, vol. 75, pp. 11-15, 2017.

[18] P. Park, W. I. Lee, and S. Y. Lee, "Auxiliary function-based integral inequalities for quadratic functions and their applications to time-delay systems," Journal of the Franklin Institute, vol. 352, no. 4, pp. 1378-1396, 2015.

[19] C.-K. Zhang, Y. He, L. Jiang, M. Wu, and H.-B. Zeng, "Stability analysis of systems with time-varying delay via relaxed integral inequalities," Systems \& Control Letters, vol. 92, pp. 52-61, 2016.

[20] K. Liu, A. Seuret, and Y. Xia, "Stability analysis of systems with time-varying delays via the second-order Bessel-Legendre inequality," Automatica, vol. 76, pp. 138-142, 2017.

[21] Z. Li, H. Yan, H. Zhang, X. Zhan, and C. Huang, "Improved inequality-based functions approach for stability analysis of time delay system," Automatica, vol. 108, Article ID 108416, 2019.

[22] J. Tian, Z. Ren, and S. Zhong, "A new integral inequality and application to stability of time-delay systems," Applied Mathematics Letters, vol. 101, Article ID 106058, 2020.

[23] H. Chen, Y. Zhang, and Y. Zhao, "Stability analysis for uncertain neutral systems with discrete and distributed delays," Applied Mathematics and Computation, vol. 218, no. 23, pp. 11351-11361, 2012.

[24] X.-G. Li and X.-J. Zhu, "Stability analysis of neutral systems with distributed delays," Automatica, vol. 44, no. 8, pp. 2197-2201, 2008.

[25] M. Syed Ali, "Stability analysis of Markovian Jumping stochastic Cohen-Grossberg neural networks with discrete and distributed time varying delays," Chinese Physics B, vol. 6, Article ID 060702, 2014.

[26] M. Syed Ali and R. Saravanakumar, "Novel delay-dependent robust $H_{\infty}$ control of uncertain systems with distributed time-varying delays," Applied Mathematics and Computation, vol. 249, pp. 510-520, 2014.

[27] S. Xu and T. Chen, "An LMI approach to the $H_{\infty}$ filter design for uncertain systems with distributed delays," IEEE Transactions on Circuits and Systems II: Express Briefs, vol. 51, no. 4, pp. 195-201, 2004.

[28] D. Yue, E. Tian, and Y. Zhang, "Delay-distribution-dependent stability and stabilization of T-S fuzzy systems with probabilistic interval delay," IEEE Transactions on Systems, Man, and Cybernetics-Part B, vol. 39, pp. 503-516, 2009. 